\title{
JORNADA PARCIAL: ¿ESPECIAL, GENERAL O AMBAS?
}

\author{
María Cristina Gajardo Harboe ${ }^{*}$ \\ Universidad de Chile
}

\section{INTRODUCCIÓN}

Se ha venido resolviendo en sede judicial, que en caso de convenirse una jornada semanal de trabajo superior a 30 horas e inferior a 45 horas, existe obligación legal de remunerar dicho tiempo con el ingreso mínimo mensual, y que todo trabajo que se encuentre en dicho rango de horas, es decir, entre 31 y 44 horas, constituiría jornada ordinaria, desde que sólo se permiten jornadas parciales de hasta 30 horas por semana. Este criterio admite una sola jornada parcial, la regulada por los artículos 40 bis a 40 bis D del Código del Trabajo, para los efectos de la exigencia de retribuir con el ingreso mínimo mensual proporcional a la jornada de trabajo.

Los preceptos legales relacionados son los artículos 22, 40 bis, 42 letra a) y 44 inciso tercero, todos del Código del Trabajo, y en torno a ellos existen argumentos técnicos relevantes que permiten concluir:

\begin{abstract}
"que son jornadas parciales aquellas inferiores a 45 horas semanales de trabajo; que los artículos 40 bis a 40 bis D del Código del Trabajo, introducidos por la Ley $\mathrm{N}^{\circ} 19.759$, establecen un estatuto especial aplicable sólo a las jornadas parciales de hasta 30 horas semanales; y que tratándose de jornadas parciales entre 31 y 44 horas, no rigen los artículos 40 bis a 40 bis D citados, aplicándose respecto de ellas las reglas comunes que están vigentes, entre las que se encuentra el artículo 44 inciso tercero del Código del Trabajo, que establece: "El monto mensual del sueldo no podrá ser inferior al ingreso mínimo mensual. Si se convinieren jornadas parciales de trabajo, la remuneración no podrá ser inferior a la mínima vigente, proporcionalmente calculada en relación con la jornada ordinaria de trabajo".
\end{abstract}

En el presente trabajo nos referiremos a estos argumentos técnicos relevantes, en base a los cuales sostenemos que en el modelo vigente de jornada parcial del Código del Trabajo, está, por una parte la regulación de una jornada parcial especial entre los artículos 40 bis a 40 bis D, y por otra, la regulación de la jornada parcial general en los artículos 22 y 44 inciso tercero, todos del Código del Trabajo.

\footnotetext{
* Abogado. Profesora de Derecho del Trabajo y de la Seguridad Social. Facultad de Derecho, Universidad de Chile. Mis agradecimientos al Ayudante de cátedra Roberto Cerón Reyes, quien colaboró en la búsqueda de antecedentes técnicos.
} 


\section{ORÍGENES DE LA REGULACIÓN. HISTORIA FIDEDIGNA DE LAS NORMAS}

1.1 La Ley No 4.053, de 29 de SEPTIEMbre de 1924, SObRe CONTRATO DE TRABAjo ObReRO, ESTABLECIÓ:

"De la duración del trabajo

Art. 11. La duración del trabajo ordinario efectivo de cada obrero, de uno u otro sexo, no podrá exceder de ocho horas por día o de cuarenta y ocho horas por semana.

Art. 13. No obstante lo dispuesto en los artículos 11 y 12, los obreros podrán estipular por salarios complementarios, mayor número de horas efectivas de trabajo, con tal que no excedan de diez horas al día y que estén interrumpidas por un descanso no menor de diez horas entre una y otra jornada...”.

Observación: esta ley no trató el tema de las jornadas parciales.

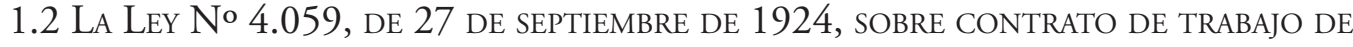
EMPLEADOS PARTICULARES, SEÑALÓ:

"De la duración del trabajo

Art. 10. La duración del trabajo de los empleados será hasta de cuarenta y ocho horas semanales, que se distribuirán en jornadas hasta de doce horas.

En casos extraordinarios, y a petición escrita del patrón o del empleado, podrá extenderse hasta cincuenta y seis horas semanales, debiendo el patrón abonar a los empleados por el trabajo extraordinario, un monto proporcional de los emolumentos, con un cincuenta por ciento (50\%) de recargo.

La duración de la jornada podrá ser de cincuenta y seis horas para los empleados de telégrafos y teléfonos, en aquellas oficinas en que el movimiento sea escaso, o sólo se lleve a cabo en determinadas horas del día."

Observación: la norma reconoció el criterio de la proporcionalidad, pero para una mayor jornada.

\subsection{El DL 670 de 1974, sobre "Reajuste, a contar del 1 de octubre de 1974, de} Sueldos del Sector Público y Privado”

El artículo 8 , incisos $1^{\circ}$ y $2^{\circ}$, de este decreto ley estableció lo siguiente:

"El ingreso mínimo mensual de los trabajadores que cumplan jornada ordinaria completa en la institución, empresa, actividad o faena, será de $\$ 2.000$, el que será imponible.

En los casos de trabajadores que presten servicios por hora o en jornada parcial de trabajo, el ingreso mínimo se determinará en proporción a la cantidad establecida en el inciso anterior".

Observación: conforme a este precepto, a una jornada ordinaria inferior a la legal correspondía pagar un ingreso mínimo proporcional a la menor jornada convenida, consagrándose por primera vez de modo expreso el criterio de la proporcionalidad del ingreso mínimo para jornadas de carácter parcial. 


\subsection{El DL 2200 DE 1978 y SU HISTORIA FIDEDIGNA}

El artículo 52 inciso $3^{\circ}$ del DL 2200, incorporó al articulado del Código del Trabajo lo siguiente:

"El monto mensual de la remuneración no podrá ser inferior al ingreso mínimo mensual. Si se convinieren jornadas parciales de trabajo, la remuneración no podrá ser inferior a la mínima vigente, proporcionalmente calculada en relación con la jornada ordinaria de trabajo".

Observación: siguió la línea establecida por el artículo 8 del DL 670 de 1974.

\subsection{LA LEY No 19.759, DE 5 DE OCTUBRE DE 2001 y SU HiSTORIA FIDEDIGNA ${ }^{1}$}

Se extrajeron los siguientes párrafos para graficar la intención del legislador en relación al fortalecimiento del empleo a tiempo parcial:

\section{A) Mensaje}

I Objetivos de la iniciativa:

2. Posibilitar relaciones laborales armónicas en un mundo globalizado.

"En segundo lugar, las normas propuestas buscan posibilitar relaciones laborales armónicas, que permitan a las partes de la relación laboral enfrentar desafíos que impone una economía abierta en un mundo globalizado, en el que la rapidez de los cambios viene dado por la fuerte incidencia de la introducción de la tecnología a las relaciones de producción y los consecuentes cambios a la organización del trabajo..." (p. 2).

\section{Nuevas modalidades de contratación}

"Por otra parte, conscientes de las transformaciones en el mercado de trabajo, y principalmente en la estructura del empleo y de la organización del trabajo, se ha estimado necesario incorporar a la legislación del trabajo, diversas fórmulas contractuales de promoción del empleo..." (p. 5).

En razón de ello se articulan varios puntos, entre los que se encuentra la flexibilización de la jornada laboral.

\section{B) Senado:}

A propósito de la exposición del señor Ministro del Trabajo y Previsión Social:

"Por otra parte, debe fortalecerse la capacidad de adaptación de nuestras empresas a una economía cada vez más abierta y sujeta a la competencia, cuestión que todas las señales muestran se irá acrecentando, y, por eso, se viene innovando en materia de jornada laboral y de contrato. Esto, precisó, se quiere hacer cumpliendo una doble función: primero, mejorar la capacidad de las empresas para adaptarse y, segundo, permitir la incorporación de grupos más vulnerables en los mercados de trabajo, creando, por estas dos vías, más oportunidades de empleo que den 
María Cristina Gajardo HarboelJornada parcial: ¡especial, general o ambas?

lugar a derechos laborales universales de todo trabajador, como son los previsionales, los relativos a seguros de accidentes en el trabajo, vacaciones, etcétera...” (p. 43).

A propósito de la discusión en general en el Senado:

Intervención del senador Cordero:

“...Por otra parte, la forma de pactar las horas extraordinarias; la rigidez establecida para pactar las jornadas especiales, quedando esta facultad entregada completamente a la Dirección del Trabajo; la limitación injustificada de la jornada parcial; el pago de compensación por la transformación del contrato de jornada completa a parcial; el pago extraordinario del tiempo destinado a la capacitación de los trabajadores, y el pago de un bono por la contratación de reemplazantes en una huelga, constituyen tópicos que restan conveniencias a estas figuras, incrementan los costos del empleador y traen, por consecuencia, un impacto negativo en la competitividad de las empresas..." (p. 185).

A propósito del segundo informe de la Comisión de Trabajo y Previsión Social:

Indicación número 11 relativa a la Jornada Parcial de Trabajo. Fue objeto de varias indicaciones. Cabe rescatar las siguientes:

a) Indicación № 54, de los HH. Senadores señor Lavandero, Ruiz De Giorgio y Ruiz-Esquide, es para suprimirlo. En la discusión el senador Ruiz de Giorgio expresó que: "ya que el Código del Trabajo permite las jornadas parciales, no ve la razón para aprobar el № 11" (p. 272). Se transcribe parte de la discusión:

"La señora Directora del Trabajo -María Ester Feres-, explicó la propuesta del Ejecutivo, señalando que es cierto que hoy día nada impide que los trabajadores, de acuerdo al artículo 22 del Código, pacten jornadas parciales, en tanto el límite de 48 horas es el límite máximo de la jornada semanal. Y existe otra norma que tiene que ver con la remuneración mínima que también establece la regla de la proporcionalidad; sin embargo, esta propuesta del Ejecutivo hay que entenderla como una figura promocional del trabajo a tiempo parcial, en términos de que respecto de grupos de trabajadores que puedan eventualmente estar fuera del mercado de trabajo haya algún atractivo especial para su contratación. El primero es una norma de flexibilidad de jornada que no existe en nuestra legislación y que ha sido establecida jurisprudencialmente por la Dirección del Trabajo, respecto de que estando claro para las partes el inicio y término de la jornada con una anticipación debida, en este caso quince días, las partes pueden variar la hora de ingreso y de término de la jornada parcial; por lo tanto, aparece por ley, una flexibilidad que no está dada para el conjunto de los contratos.

En segundo lugar, viene una norma proporcional que falta en el Código, porque si bien todos los beneficios de la jornada de tiempo parcial tienden a ser proporcionales, hay uno, relativo a la gratificación, en el cual no existe la proporcionalidad. Entonces el segundo elemento proporcional es establecer, como regla general, también una proporcionalidad en materia de gratificaciones. Y el tercero, que es más discutido por las distintas indicaciones, tiene que ver con la posibilidad de modificar las cláusulas de un contrato a tiempo completo por cláusulas a tiempo parcial sin necesidad de finiquitar la relación laboral. Porque eventualmente, 
si alguien tiene jornada completa, finiquita y vuelve a contratar en otras condicione; lo que propone el Ejecutivo es que sin perder esa relación se baje proporcionalmente el costo indemnizatorio. Hay un objetivo general de promocionar la idea de la jornada a tiempo parcial" (p. 272).

La indicación fue rechazada.

b) Indicación $\mathrm{N}^{0}$ 59, del H. Senador señor Vega, es para sustituir el artículo 40-A propuesto por el siguiente: "Artículo 40-A.- Se podrán pactar contratos de trabajo con jornada parcial, considerándose afectos a la normativa del presente párrafo, aquellos que se han convenido una jornada de trabajo inferior a la jornada ordinaria a que se refiere el artículo 22".

La indicación fue rechazada y tampoco fue discutida.

C) Cámara de Diputados:

Informe de la Comisión de Trabajo y Seguridad Social. El artículo 40 bis (Jornada Parcial) no fue objeto de ninguna indicación. En esta materia lo que se discutió fue el tema del cálculo de la indemnización del trabajador al término de la relación de trabajo (artículo 40 bis D).

\subsection{La Ley No 20.281, de 21 de julio de 2008, Que modifica el Código del Trabajo} EN MATERIA DE SALARIOS BASE, Y SU HISTORIA FIDEDIGNA ${ }^{2}$

Existen antecedentes explícitos que avalan un pago proporcional al sueldo mínimo a las jornadas parciales superiores a 30 horas y menores a 45 horas.

Las razones que sustentan esta posición son las siguientes:

1) El mensaje del Ejecutivo propone "delimitar" y "diferenciar" en el concepto de remuneración los componentes fijos de los variables. Así, asocia al primero con el tiempo que el trabajador está a disposición del empleador para prestar los servicios acordados en el contrato de trabajo, es decir, lo relaciona con el concepto de jornada de trabajo. El segundo lo identifica con la productividad del trabajador. De este modo:

“...los elementos de la prestación de los servicios desde la perspectiva de las remuneraciones, son dos: el cumplimiento de la jornada y la productividad del trabajador. De esta forma, no cabe sino concluir que el sueldo o estipendio fijo que percibe el trabajador, corresponde al tiempo de prestación de sus servicios en la empresa, es decir a la remuneración que tiene como correspondencia la prestación de los servicios en una jornada ordinaria pactada, dejando a otros elementos la compensación por la productividad del trabajador. Dicho preliminarmente, si el sobresueldo es la contraprestación por una mayor jornada que la ordinaria (jornada extraordinaria), forzoso es concluir que el sueldo es la contraprestación por los servicios dentro de la jornada

Véase www.bcn.cl/histley/lfs/hdl-20281/HL20281.pdf 
María Cristina Gajardo Harboe/Jornada parcial: ¡especial, general o ambas?

ordinaria de trabajo. Expresado de otra forma: el sobresueldo hace sentido a la jornada extraordinaria, tanto como el sueldo hace sentido a la jornada ordinaria" (p. 6).

De esta manera el Ejecutivo relaciona una jornada ordinaria de trabajo con el pago del sueldo, remuneración que con este proyecto de ley se asimiló al Ingreso Mínimo Mensual. También engarza con el criterio de proporcionalidad en el pago del Ingreso Mínimo Mensual cuando la jornada es parcial. En este sentido:

"Ello se encuentra en íntima correspondencia con el concepto doctrinario de Ingreso Mínimo Mensual, que ubica una cantidad mínima de compensación al trabajador por sus servicios, y que tiene por objeto proteger el ingreso al mercado laboral del trabajador menos calificado y al más vulnerable, cuya primera obligación es cumplir con la jornada ordinaria de trabajo, percibiendo por ello una remuneración que no puede ser inferior a la que la ley determine.

El ingreso mínimo no tiene como objeto dar una señal económica de proyección del gasto familiar, sino que es un mínimo tolerable para una sociedad, al aceptar que una persona se incorpore al mercado laboral cumpliendo a lo menos una jornada ordinaria de trabajo.

La interpretación antedicha, se refuerza al analizar la continuación del inciso tercero del artículo 44, que señala: si se convinieren jornadas parciales de trabajo, la remuneración no podrá ser inferior a la mínima vigente, proporcionalmente calculada en relación con la jornada ordinaria de trabajo.

Es decir, si el salario mínimo se paga proporcional a la jornada de trabajo pactada en el contrato, es evidente que el elemento de la prestación de los servicios que remunera el IMM, es el de la permanencia del trabajador a disposición del empleador, prestando servicios en una jornada ordinaria..." (p. 6).

2) La idea anterior se ve reforzada en los "Contenidos de la Iniciativa". En efecto, uno de los puntos es la asimilación del sueldo base al ingreso mínimo mensual: "Dado que el sueldo base remunera, como se ha expresado, la relación laboral desarrollada en un régimen de dependencia y subordinación por un trabajador en a lo menos una jornada ordinaria legal, se establece que dicho sueldo base no puede ser inferior a un Ingreso Mínimo Mensual". (p. 7)

3) Estas orientaciones se repiten a lo largo de toda la tramitación del proyecto. Las citamos a continuación:

A) Cámara de Diputados:

- Informe Comisión de Trabajo: pp. 10, 13

- Discusión en sala: pp. 20, 21

B) Senado:

- Informe de Comisión de Trabajo: pp. 29, 30, 31, 33, 34 


\section{EL ELEMENTO LÓGICO EN LA INTERPRETACIÓN}

1) Cabe preguntarse si es posible que el legislador estipule que a quien se le remunere con el ingreso mínimo y trabaje 31, 32, 33, 34, 35, 36, 37, 38, 39, 40, 41, 42, 43 ó 44 horas, se le entregue la misma suma de dinero que quien laboró la jornada ordinaria de 45 horas semanales.

Si esta era la intención del legislador, atendido el carácter de normas de orden público, aquél debió dejar expresamente asentado que quien está remunerado con el ingreso mínimo mensual lo debe percibir completo, sea que trabajo sólo una jornada parcial de 31 a 44 horas semanales. Y claramente la ley no estableció tal cosa.

2) Las jornadas parciales superiores a 30 e inferiores a 45 horas semanales, por lógica, han de ser parciales, desde que la ordinaria es de 45 horas semanales, y al ser distintas, de menos horas de trabajo, no pueden ser tratadas remuneracionalmente de la misma manera que una de mayor tiempo de trabajo, pues ello también contraviene el artículo 41 del Código del Trabajo, que entiende a la remuneración como la contraprestación en dinero y las adicionales en especie avaluables en dinero, que el trabajador percibe "por causa del contrato de trabajo", desde que quien trabaja 45 horas y es remunerado con el ingreso mínimo, en el hecho percibiría una suma inferior que quien trabaja menos y es remunerado con el mismo mínimo, pues éste recibirá igual cantidad.

Lo anterior llevará a que en el futuro las jornadas que los empleadores contraten, llegarán hasta las 30 horas y el resto será contratado por 45 horas semanales, aunque se le necesite por menos, pues si remunera el empleador con el ingreso mínimo, deberá pagar la misma cantidad de dinero a quien asiste 31 hora semanales y a quien lo hace por 45 horas.

No ha podido ser ésta la intención del legislador, más aún cuando por la incorporación del artículo 40 bis $\mathrm{A}$, inciso $2^{\circ}$, del Código del Trabajo, se mantuvo vigente de todas formas el artículo 44 , inciso $3^{\circ}$, del mismo código, precepto este último que es de aplicación general.

3) Pretender aplicar la exigencia del ingreso mínimo mensual a personas que laboran entre 31 y 44 horas por semana, abarca universos de trabajadores que de acuerdo a la experiencia suelen trabajar en dicho régimen horario, como son los trabajadores de casa particular y el personal docente regido por la Ley $\mathrm{N}^{\circ} 19.070$.

En los trabajadores de casa particular, rige el criterio de la proporcionalidad en el ingreso mínimo para una jornada inferior a las 45 horas, como es el caso de quien asiste tres días a la semana con una jornada diaria de 12 horas, conforme lo autoriza el artículo 149 del Código del Trabajo, en relación con el artículo 151 inciso $3^{\circ}$ del mismo código, que vuelve sobre la proporcionalidad al establecer que "los trabajadores que no vivan en la casa del empleador y se desempeñen a jornadas parciales o presten servicios sólo algunos días a la semana, tendrán derecho a una remuneración mínima no inferior a la referida en el inciso anterior, proporcionalmente calculada en relación con la jornada o los días de trabajo.

Similar es la situación de los docentes regidos por la Ley $\mathrm{N}^{\circ} 19.070$, cuyos artículos 68, 69, 78,79 y 80 , establecen que cuando la jornada de trabajo contratada fuere inferior a 44 horas semanales, el máximo de clases quedará determinado por la proporción respetiva. 
María Cristina Gajardo HarboelJornada parcial: ¡especial, general o ambas?

4) El criterio de la proporcionalidad es un paradigma presente a lo largo de todo el Código del Trabajo, como en los casos descritos en el número 3) precedente, en el feriado (artículo 73 inciso $3^{\circ}$ ) y en las gratificaciones legales (artículo 52).

No existe razón ni explicación lógica para que sólo en el caso de la jornada parcial que va entre 31 y 44 horas semanales, no se aplique la proporcionalidad que se viene refiriendo.

\section{INTERPRETACIÓN POR VÍA DE AUTORIDAD}

En sede administrativa, la Dirección del Trabajo ha dictaminado lo siguiente:

1) A propósito de las reformas introducidas al artículo 42, letra a) del Código del Trabajo por la Ley $\mathrm{N}^{\circ} 20.281$ :

"Del análisis conjunto de los preceptos legales citados se infiere, en primer término, que el legislador ha establecido un nuevo concepto de sueldo, asimilándolo al sueldo base, precisando que tal estipendio es de carácter obligatorio y no podrá ser inferior al valor fijado para un ingreso mínimo mensual, cuando se ha convenido la jornada ordinaria máxima legal prevista en el inciso $1^{\circ}$ del artículo 22 del Código del Trabajo, vale decir, 45 horas semanales", y que "Se infiere asimismo, que tratándose de jornadas parciales de trabajo, el sueldo pactado no podrá ser inferior al ingreso mínimo vigente, proporcionalmente calculado en relación a dicha jornada ordinaria máxima"’.

2) Respecto del Estatuto Docente, se ha sostenido que es procedente remunerar a un docente que labora 33 horas semanales con el ingreso mínimo remuneracional determinado en proporción a dicha jornada ${ }^{4}$.

También que en el caso de los profesionales de la educación del sector municipal, dentro de los cuales quedan comprendidos aquellos docentes que prestan servicios en establecimientos educacionales dependientes de las Corporaciones Municipales, se infiere que el legislador ha establecido para los mismos una Remuneración Básica Mínima Nacional, que se determina multiplicando el valor mínimo de la hora cronológica por el número de horas contratadas con el docente ${ }^{5}$.

\section{INTERPRETACIÓN JUDICIAL}

A la fecha de envío a impresión del presente trabajo, la Excma. Corte Suprema aún no había unificado jurisprudencia sobre el punto de derecho que planteamos, observándose dos líneas muy concretas de razonamiento a nivel de Cortes de Apelaciones.

Es así como una línea de razonamiento considera la tesis de remunerar con el ingreso mínimo mensual, aquellas jornadas parciales comprendidas entre las 31 y las 44 horas semanales, y

\footnotetext{
Dirección del Trabajo. Ordinarios 3152/063 de 25 de julio de 2008 y 2212/036 de 8 de junio de 2009 .

Dirección del Trabajo. Ordinario 4550/077 de 19 de octubre de 2012.

Dirección del Trabajo. Ordinario 69/02 de 7 de enero de 2013.
} 
sus argumentaciones se reúnen en la Sentencia de fecha 9 de enero de 2013, dictada por la Excma. Corte Suprema en causa Rol 5305.2012 "Aguilar y otros con Emergia Contact Center Ltda..

La otra línea de razonamiento observada, y que sostiene la tesis de la proporcionalidad del artículo 44, inciso $3^{\circ}$, del Código del Trabajo, está contenida en la Sentencia de fecha 11 de abril de 2012, dictada por la Iltma. Corte de Apelaciones de Santiago en causa Rol 1124-2011 "Silva y otros con Emergia Contact Center SL Chile Ltda...

\section{INTERPRETACIÓN DOCTRINAL}

La doctrina nacional tradicional es uniforme. Baste la cita de tres connotados tratadistas. Para Patricio Novoa y William Thayer "con la expresión "jornada ordinaria de trabajo" nos referimos a esta jornada de 45 horas semanales, pues existen jornadas especiales; reducidas o prolongadas; las que representan, en uno y otro sentido, excepciones, por razones o motivos justificados, a la jornada normal" 6 . Francisco Walker reitera que "La jornada ordinaria de trabajo no excederá de 48 horas semanales hasta el 31 de diciembre de 2004 y de 45 horas semanales desde el 1 de enero de 2005 en adelante..."

En fecha más reciente, Lautaro Fariña Quezada ha dicho que "el sueldo o sueldo base debe siempre, a lo menos, igualarse a un ingreso mínimo mensual o la proporción que corresponda según la reducción de la jornada de trabajo.”

\section{OTRAS NORMAS RELEVANTES}

En relación con la "justa retribución", es interesante abordar su alcance, dado que algunos fallos que están con la tesis de la jornada parcial única de los artículos 40 bis a 40 bis D del Código del Trabajo, recurren al argumento de que "el trabajo a tiempo parcial no puede ser una oportunidad para rebajar los costos laborales más allá de las previsiones legislativas, para abaratarlos respecto de quienes prestan servicios por más de 30 horas semanales, porque ello importaría precarizar su situación" ${ }^{\prime \prime}$, aludiendo al justo salario.

El artículo $19 \mathrm{~N}^{\circ} 16$ de la Constitución Política de la República establece: “Toda persona tiene derecho a la libre contratación y a la libre elección del trabajo con una justa retribución".

El profesor Pedro Irureta manifiesta que el contenido exacto de la expresión constitucional debe ser vinculado con la norma, de inferior jerarquía, del artículo 44, inciso tercero, del Código del Trabajo, sobre remuneración mínima. Y de ello se deriva que no existe autonomía de las partes para fijarla, al existir un límite desde el punto de vista legal ${ }^{10}$.

Ahora bien, el límite legal establecido en el artículo 44, inciso tercero, del Código del Trabajo establece: "El monto mensual del sueldo no podrá ser inferior al ingreso mínimo mensual. Si

Novoa Fuenzalida, Patricio y Thayer Arteaga, William. Manual de Derecho del Trabajo. Tomo II. -5º edición actualizada-. Santiago, Chile: Editorial Jurídica de Chile, 2008, p. 125

Walker Errazuriz, Francisco. Derecho de las Relaciones Laborales. Santiago, Chile: Editorial Universitaria, 2003, p. 305.

FariN̄a Quezada, Lautaro. Regulación del sueldo en el Código del Trabajo. Santiago, Chile: LegalPublishing, 2009, p. 80.

Sentencia de 16 de enero de 2013, Corte de Apelaciones de Santiago, Reforma Laboral N¹564-2012, considerando séptimo.

Irureta Uriarte, Pedro. Constitución y Orden Público Laboral. Un análisis del art. $19 \mathrm{~N}^{\circ} 16$ de la Constitución Politica Chilena. Santiago, Chile: Colección de Investigaciones Jurídicas, Universidad Alberto Hurtado, 2006, p. 70. 
María Cristina Gajardo HarboelJornada parcial: ¡especial, general o ambas?

se convinieren jornadas parciales de trabajo, la remuneración no podrá ser inferior a la mínima vigente, proporcionalmente calculada en relación con la jornada ordinaria de trabajo."

En consecuencia, la justa retribución ha de ser entendida como aquella equivalente a un sueldo de un monto no inferior a un Ingreso Mínimo Mensual, y que en caso de jornadas parciales (cualquiera que ellas sean por debajo de las 45 horas semanales) corresponde un Ingreso Mínimo Mensual pero en una proporción inferior, en relación con la jornada ordinaria de trabajo.

\section{CONVENIOS OIT}

Revisados todos los convenios relacionados con el tema del informe, no hay nada relevante a excepción del Convenio No 175, de 1994, sobre Trabajo a Tiempo Parcial, que señala ciertas pautas de interés.

Si bien este instrumento no se encuentra ratificado por Chile, su contenido se orienta en el sentido de reconocer la proporcionalidad del ingreso mínimo como remuneración en jornadas parciales, según se destaca a continuación:

\section{ArTí́CUlo 1}

A efectos del presente Convenio:

(a) la expresión trabajador a tiempo parcial designa a todo trabajador asalariado cuya actividad laboral tiene una duración normal inferior a la de los trabajadores a tiempo completo en situación comparable;

(b) la duración normal de la actividad laboral a la que se hace referencia en el apartado a) puede ser calculada semanalmente o en promedio durante un período de empleo determinado;

(c) la expresión trabajador a tiempo completo en situación comparable se refiere al trabajador a tiempo completo que:

(i) tenga el mismo tipo de relación laboral;

(ii) efectúe o ejerza un tipo de trabajo o de profesión idéntico o similar, y

(iii) esté empleado en el mismo establecimiento o, cuando no haya ningún trabajador a tiempo completo en situación comparable en ese establecimiento, en la misma empresa o, cuando no haya ningún trabajador a tiempo completo en situación comparable en esa empresa, en la misma rama de actividad, que el trabajador a tiempo parcial de que se trate;

(d) no se considerará trabajadores a tiempo parcial a los trabajadores a tiempo completo que se encuentren en situación de desempleo parcial, es decir, aquellos que estén afectados por una reducción colectiva y temporal de la duración normal de su trabajo por motivos económicos, tecnológicos o estructurales. 


\section{ARTí́CUlo 4}

Deberán adoptarse medidas para asegurar que los trabajadores a tiempo parcial reciban la misma protección de que gozan los trabajadores a tiempo completo en situación comparable en lo relativo:

(a) al derecho de sindicación, al derecho de negociación colectiva y al derecho de actuar en calidad de representantes de los trabajadores;

(b) a la seguridad y la salud en el trabajo;

(c) a la discriminación en materia de empleo y ocupación.

Observación: no menciona jornadas parciales ni ingreso mínimo.

\section{ARTí́CUlO 5}

Deberán adoptarse medidas apropiadas a la legislación y la práctica nacionales para asegurar que los trabajadores a tiempo parcial no perciban, por el solo hecho de trabajar a tiempo parcial, un salario básico que, calculado proporcionalmente sobre una base horaria, por rendimiento o por pieza, sea inferior al salario básico, calculado por el mismo método, de los trabajadores a tiempo completo que se hallen en una situación comparable.

Observación: establece la proporcionalidad del salario mínimo como criterio.

\section{ARTí́CUlo 6}

Los regímenes de seguridad social establecidos por ley que estén ligados al ejercicio de una actividad profesional deberán adaptarse de forma que los trabajadores a tiempo parcial gocen de condiciones equivalentes a las de los trabajadores a tiempo completo en situación comparable; estas condiciones podrán determinarse de forma proporcional a la duración del tiempo de trabajo, a las cotizaciones o los ingresos, o mediante otros métodos que sean conformes a la legislación y la práctica nacionales.

Observación: refiere condiciones equivalentes, pero no "idénticas". Consagra la proporcionalidad en materia de seguridad social, en el entendido de que las prestaciones pecuniarias podrán determinarse proporcionalmente a la duración del tiempo de trabajo o a los ingresos.

\section{CONCLUSIONES}

A partir de los antecedentes técnicos ya presentados es posible sostener lo siguiente:

1) El criterio de la proporcionalidad en el Ingreso Mínimo Mensual respecto de jornadas parciales de trabajo, se consagró por primera vez en el DL 670 de 1974 y se ha mantenido hasta hoy vigente, a través del artículo 44, inciso $3^{\circ}$, del Código del Trabajo. 
María Cristina Gajardo Harboe/Jornada parcial: ¡especial, general o ambas?

La modificación introducida por la Ley $\mathrm{N}^{\circ} 19.759$ sólo tuvo por fin incorporar los artículos 40 bis a 40 bis $\mathrm{D}$ al Código del Trabajo como un estatuto especial, y como una forma de incentivar el empleo, pero no derogó ni eliminó las normas generales sobre jornada parcial, entendida como aquella inferior a las 45 horas semanales de trabajo, y de ello da cuenta la historia fidedigna del establecimiento de estas normas.

2) En cuanto al Ingreso Mínimo Mensual, las modificaciones que introdujo la Ley $\mathrm{N}^{\circ} 20.281$ al Código del Trabajo, también de acuerdo a la historia fidedigna de su establecimiento, refieren que la exigencia de retribuir con un sueldo de un monto no inferior a un Ingreso Mínimo Mensual, lo es para jornadas ordinarias de trabajo, y que si éstas son parciales, reconocen explícitamente el criterio de la proporcionalidad respecto del Ingreso Mínimo Mensual.

3) Por ello, es fundamental atender al elemento histórico de interpretación de la ley -artículo 19 inciso $2^{\circ}$ del Código Civil-, con el cual se obtiene una interpretación de jornada parcial y su relación con el Ingreso Mínimo Mensual que admite la jornada parcial en sus especies de "especial" y "general". El alcance de este elemento histórico es descrito por Ducci: “...el precepto nos dice para qué puede recurrirse a la historia fidedigna del establecimiento de la ley, y ello es para determinar su razón o espíritu, los de la ley...Así que lo que se busca es la intención o el espíritu objetivo de la ley..."11.

En relación con este elemento histórico, es posible afirmar que es errado el criterio de considerar como única jornada parcial de trabajo la regulada en los artículos 40 bis a 40 bis D del Código del Trabajo. El error descansa en un desconocimiento de la historia de la ley No 19.759, que señaló expresamente los fines promocionales de la jornada parcial regulada en el Código, y que en ningún momento la instituyó como única, así como de la ley No 20.281, que supuso el pago del sueldo base -equivalente al Ingreso Mínimo Mensual- en relación a una jornada ordinaria de trabajo - 45 horas-, ratificando el criterio de "proporcionalidad" en el pago de jornadas inferiores a la ordinaria.

4) En un análisis lógico, se arriba a la misma conclusión, pues la tendencia general en nuestra legislación es aplicar la proporcionalidad, en la medida que exista un menor tiempo de trabajo, sea en jornada, feriado, gratificaciones o sueldo base. De otra forma, no se comprende el sentido del artículo 44, inciso $3^{\circ}$, del Código del Trabajo, ni las razones por las cuales el criterio de la proporcionalidad en jornadas parciales se mantiene respecto de otros grupos de trabajadores (trabajadores de casa particular y docentes).

5) En el ámbito de la interpretación de las normas en análisis por vía de autoridad, llama la atención que la Dirección del Trabajo haya sancionado por el no pago del Ingreso Mínimo Mensual en jornadas parciales entre 31 y 44 horas (causa RIT I-237-2009 del Juzgado de Letras de Valparaíso) ${ }^{12}$, y que desde su Departamento Jurídico haya dictaminado que a una jornada parcial le corresponde un Ingreso Mínimo Mensual proporcionalmente inferior.

11 Ducci Claro, Carlos. Interpretación jurídica. -3o edición-. Santiago, Chile: Editorial Jurídica de Chile, 2006, p. 129

12 Esta es la sentencia de la jueza Ximena Cárcamo, que dio inicio a la línea jurisprudencial que reconoce una sola jornada parcial 
Nos parece que han de prevalecer los dictámenes citados en el acápite III de este informe, puesto que emanan del Departamento Jurídico de la Dirección del Trabajo.

6) En definitiva, para buscar el sentido de la ley es preciso recurrir a las normas sobre interpretación de la misma contenidas en el Código Civil, entre los artículos 19 y 24, en donde están los elementos tradicionales de la interpretación: el gramatical, el histórico, el lógico, el sistemático, y por último, el espíritu general de la legislación y la equidad natural.

Estos elementos deben ser utilizados para fijar el correcto sentido de la ley frente al caso específico, más allá de consideraciones de justicia social, pues corresponde a los tribunales de justicia resolver los casos sometidos a su conocimiento aplicando el derecho vigente, el que para el caso de las jornadas parciales inferiores a 45 horas semanales, establece que "la remuneración no podrá ser inferior a la mínima vigente, proporcionalmente calculada en relación con la jornada ordinaria de trabajo " (artículo 44, inciso $3^{\circ}$, del Código del Trabajo).

A modo de cierre, estimamos que al margen de consideraciones de orden económico y social en torno al monto que debe tener el ingreso mínimo mensual, sea para una jornada de trabajo de 45 horas semanales, sea para una proporción de ésta, nos parece que desde el punto de vista legal, el modelo vigente en nuestro Código del Trabajo consagra dos tipos de jornada parcial: la que hemos venido señalando como "especial", regulada en los artículos 40 bis a 40 bis D del citado código, y la de orden "general" o residual, que es la que siempre rigió para los casos en que se conviniera una jornada inferior a 45 horas -o 48 horas en su época- con base en los artículos 22 y 44 inciso $3^{\circ}$ del Código del Trabajo, jornada esta última que no ha sido derogada por las Leyes $\mathrm{N}^{\circ} 19.759$ y N²0.281, de modo tal que se mantiene plenamente vigente.

en el Código del Trabajo. 